Revue internationale P.M.E.

Économie et gestion de la petite et moyenne entreprise

\title{
Introduction en Bourse, conduite stratégique et performance des moyennes entreprises françaises : une étude empirique
}

\section{Nazik Fadil}

Volume 18, numéro 3-4, 2005

URI : https://id.erudit.org/iderudit/1008485ar

DOI : https://doi.org/10.7202/1008485ar

Aller au sommaire du numéro

Éditeur(s)

Presses de l'Université du Québec

ISSN

0776-5436 (imprimé)

1918-9699 (numérique)

Découvrir la revue

Citer cette note

Fadil, N. (2005). Introduction en Bourse, conduite stratégique et performance des moyennes entreprises françaises : une étude empirique. Revue

internationale P.M.E., 18(3-4), 125-148. https://doi.org/10.7202/1008485ar
Résumé de l'article

L'objet de cet article est d'étudier l'effet de la cotation des moyennes entreprises françaises sur leur performance financière. Cet effet se traduit par une modification du système de gouvernance et il a, par conséquent, un impact sur la conduite stratégique du décideur. Notre objectif est d'évaluer, autant que faire se peut, cet impact en explorant brièvement les fondements théoriques qui sous-tendent la décision d'introduction en Bourse et en testant empiriquement ces propositions, grâce à une étude statistique et économétrique. 
Notes de recherche

\title{
Introduction en Bourse, conduite stratégique et performance des moyennes entreprises françaises: une étude empirique ${ }^{1}$
}

Nazik FADIL École de management de Normandie

MOTS CLÉS

\section{Bourse - Moyennes entreprises - Performance financière} Conduite stratégique - Gouvernance

\begin{abstract}
RÉSUMÉ
L'objet de cet article est d'étudier l'effet de la cotation des moyennes entreprises françaises sur leur performance financière. Cet effet se traduit par une modification du système de gouvernance et il a, par conséquent, un impact sur la conduite stratégique du décideur. Notre objectif est d'évaluer, autant que faire se peut, cet impact en explorant brièvement les fondements théoriques qui sous-tendent la décision d'introduction en Bourse et en testant empiriquement ces propositions, grâce à une étude statistique et économétrique.
\end{abstract}

\section{L'AUTEURE}

NAZIK FADIL est professeure de finance, comptabilité et contrôle de gestion à l'École de management de Normandie. Ses travaux de recherche portent sur la finance et la gouvernance au sein des PME et s'intéressent plus particulièrement à la cotation en Bourse des PME. Elle est membre de l'AIREPME et chercheure au CIME à l'IAE de Caen, Basse-Normandie. Adresse: 30, rue Richelieu, 76087 Le Havre Cédex, France. Téléphone: 33232927082. Courriel:<n.fadil@esc-lehavre.fr>.

1. L'auteure tient à remercier tous ceux qui ont contribué, par leurs précieux conseils, à la réalisation de cet article, notamment: les professeurs J.C. Papillon, H. Alexandre, D. Folus, monsieur A. Karem, madame N. Bossard et les évaluateurs de la revue. Naturellement, les erreurs éventuelles restent de son entière responsabilité.

(C) 2005 - Presses de l'Université du Québec

Édifice Le Delta I, 2875, boul. Laurier, bureau 450, Sainte-Foy, Québec G1V 2M2 • Tél.: (418) 657-4399 - www.puq.ca

Tiré de: Revue internationale P.M.E., vol. 18, nos $3-4$, sous la direction de Louis Raymond - PME1803N

Tous droits de reproduction, de traduction et d'adaptation réservés 


\begin{abstract}
The objective of this article is to study the effect of the stock exchange entry of medium-sized French companies on their financial performance. This effect operates through a modification of the governance system and thus has an impact on the strategic behaviour of the decision-maker. Our objective is to evaluate this impact, as far as possible, by briefly seeking the theoretical bases which underlie the decision to enter the stock market, and by testing these proposals empirically, through a statistical and econometric study.

\section{RESUMEN}

El objetivo de este artículo es estudiar el efecto de la cotizaciòn de bolsa de las medianas empresas en su resultado financiero. Este efecto se hace a través de una modificación del sistema del gobierno y entonces a través de un impacto en el comportamiento estratégico del responsable. Nuestro objetivo es evaluar este impacto, en lo posible, buscando brevemente las bases teóricas que explican la decisión de ingresar en la bolsa, y probando estas ofertas empiricamente, con un estudio estadístico y econométrico.
\end{abstract}

\title{
ZUSAMMENFASSUNG
}

Der Artikel soll die Auswirkungen einer Börsenkotierung von mittelgrossen französischen Unternehmen auf ihre finanzielle Leistungsfähigkeit untersuchen. Mit einem IPO ist eine Änderung des Managementsystems und somit ein Wandel in der strategischen Führung verbunden. Ziel ist es, die Auswirkungen die eine Börsenkotierung mit sich bringt mit Hilfe der theoretischen Konzepte abzuschätzen. Die getroffenen Einschätzungen werden empirisch (durch eine statistische und ökonometrische Studie) getestet.

\section{Introduction}

La décision de transformer une société fermée (non cotée) en société ouverte (cotée) signifie non seulement un apport immédiat en capital net, mais aussi un changement positif et permanent par rapport à l'environnement externe et financier de l'entreprise. L'accès au financement public semble également constituer une première étape en vue de la croissance et de la maturité de la PME [...] Il ressort nettement que l'expérience au financement public se révèle très positive (p. 119).

C'est en ces termes que Desroches et Jog (1991) concluaient leur enquête sur la PME et le financement par le grand public. Il apparaît ainsi que la Bourse constitue un moyen propice au développement et à la croissance de la moyenne entreprise. En effet, l'introduction en Bourse assure une rentrée de fonds importante, accroît la notoriété et le pouvoir de négociation avec les divers partenaires (Levasseur et Quintart, 1998), augmente la mobilité du capital (Saada, 1996), contrôle les 
dirigeants (Daigne et Joly, 1986) et favorise les stratégies de croissance externe (Levasseur et Quintart, 1998). Cela étant dit, elle n'est toutefois pas sans contrepartie: la cotation nécessite temps et énergie et représente un coût important pour une entreprise de dimension moyenne (Desjardin, 1999). En outre, elle astreint à distribuer régulièrement des dividendes ${ }^{2}$ et à répondre à des exigences de transparence et de conformité aux prévisions. Faute de quoi et à certaines conditions, l'entreprise pourrait faire l'objet d'une offre publique d'achat ou OPA (Saada, 1996).

Ainsi, il en découle une modification des données de l'environnement de la moyenne entreprise, des avantages et des contraintes qui ne sont pas sans influencer les choix stratégiques des dirigeants. En se référant notamment à la théorie de l'agence, l'influence de la discipline du marché financier inciterait les dirigeants à prendre des décisions allant dans le sens des intérêts des actionnaires. Ces décisions pourraient être différentes en l'absence de cotation. Quel en serait alors l'impact sur la performance?

Afin d'apporter quelques éléments de réponse, nous avons réalisé une étude empirique portant sur un échantillon de moyennes entreprises cotées. La première partie du présent article expose le cadre théorique, la problématique et la méthodologie utilisée, tandis que la seconde a pour objet la présentation et l'analyse des résultats.

\section{Cadre théorique, problématique et méthodologie de la recherche}

Cette partie présente brièvement les bases théoriques qui sous-tendent notre questionnement et explique la démarche utilisée pour tenter d'y répondre. Nous abordons le sujet sous l'angle des théories de la gouvernance. Le marché financier constitue, en effet, un mécanisme disciplinaire des dirigeants qui n'est pas sans influencer leur conduite stratégique et leurs performances.

\subsection{La problématique à la lumière des théories de la gouvernance}

Le concept de gouvernement d'entreprise (GE) renvoie à l'influence des décisions stratégiques sur la création de valeur. Dans la mesure où la politique générale de l'entreprise est principalement déterminée par ses dirigeants, la maximisation de la valeur est placée sous leur responsabilité. Charreaux (1997) définit ainsi le GE:

2. Cela ne se prête pas à la généralité, car nombreuses sont les sociétés qui ne distribuent pas de dividendes.

Revue internationale P.M.E., vol. 18, nºs 3-4, 2005

(C) 2005 - Presses de l'Université du Québec

Édifice Le Delta I, 2875, boul. Laurier, bureau 450, Sainte-Foy, Québec G1V 2M2 - Tél.: (418) 657-4399 - www.puq.ca

Tiré de: Revue internationale P.M.E., vol. 18, nos $3-4$, sous la direction de Louis Raymond - PME1803N

Tous droits de reproduction, de traduction et d'adaptation réservés 
«Le gouvernement des entreprises recouvre l'ensemble des mécanismes qui ont pour effet de délimiter les pouvoirs et d'influencer les décisions des dirigeants, autrement dit, qui "gouvernent" leur conduite et définissent leur espace discrétionnaire.» Dans cette définition, l'élément central est le dirigeant et son espace discrétionnaire constitue la variable qui contribue à donner une justification au système de GE. En définissant cet espace discrétionnaire et en le délimitant, l'alignement des intérêts des dirigeants et des actionnaires a de plus grandes chances d'être atteint et la richesse des actionnaires d'être ainsi maximisée. Au vu de cette définition du gouvernement d'entreprise centrée sur la notion de latitude discrétionnaire des dirigeants et du postulat selon lequel le dirigeant joue un rôle central dans le processus de création de valeur, l'analyse du dirigeant mérite une attention particulière.

La théorie de l'agence considère que les dirigeants sont les agents des actionnaires au sein de l'entreprise et qu'ils sont censés gérer l'entreprise dans l'intérêt des actionnaires (Jensen et Meckling, 1976). Or, les agents et les principaux possèdent des fonctions d'utilité différentes et agissent de façon à maximiser leur utilité respective (Ross, 1973). Le conflit d'agence conduit à l'hypothèse d'un opportunisme des dirigeants qui peut se concrétiser de diverses manières:

- La recherche de la croissance: étant donné que leur rémunération dépend de la taille de l'entreprise, les dirigeants ont tendance à privilégier l'objectif de croissance au détriment de la rentabilité, afin de maximiser la valeur de leur capital humain.

- La diversification: contrairement aux actionnaires qui peuvent facilement diversifier le risque de leur portefeuille, les dirigeants, ayant investi tout leur capital humain, sont amenés à diversifier les activités de l'entreprise, fût-ce au détriment des intérêts des actionnaires. De ce fait, les acquisitions sont d'autant plus diversifiées que la richesse des dirigeants est investie dans l'entreprise (May, 1995).

- L'enracinement: cette thèse soutenue par Jensen et Meckling (1976) stipule que plus le pourcentage détenu par les dirigeants est important, plus l'écart par rapport à l'objectif traditionnel de maximisation de la valeur est faible. Shleifer et Vishny (1989) ont proposé une autre conception de l'enracinement des dirigeants basée sur la réalisation d'investissements spécifiques qui rendent coûteux leur remplacement et leur permettent d'obtenir des rémunérations plus élevées, de plus grands avantages et plus de latitude pour déterminer la stratégie de l'entreprise. Deux perspectives sur l'enracinement des dirigeants peuvent être évoquées: 1) une perspective favorable: la réalisation par les dirigeants d'investissements spécifiques à leur compétence leur permettra de dégager des rentes dont les actionnaires et les différents partenaires seront indirectement bénéficiaires (Castanias

Revue internationale P.M.E., vol. 18, $\mathrm{n}^{\text {os }} 3-4,2005$

(C) 2005 - Presses de l'Université du Québec

Édifice Le Delta I, 2875, boul. Laurier, bureau 450, Sainte-Foy, Québec G1V 2M2 - Tél.: (418) 657-4399 - www.puq.ca

Tiré de: Revue internationale P.M.E., vol. 18 , nos $3-4$, sous la direction de Louis Raymond - PME1803N

Tous droits de reproduction, de traduction et d'adaptation réservés 
et Helfat, 1992); 2) une perspective mixte: l'enracinement des dirigeants leur permet d'élargir leur vision sur le long terme et de se dégager des pressions «court-termistes» (Pacquerot, 1997).

Afin de lutter contre ces déviations, le gouvernement d'entreprise met en place des leviers d'alignement du comportement des dirigeants. Jensen (1993) distingue quatre forces venant atténuer les divergences d'intérêt entre principal et agent: le contrôle interne (conseil d'administration, modes de rémunération, marché du travail), le marché des biens et services, les organismes de contrôle et de régulation des marchés financiers, le marché financier et le marché des fusionsacquisitions. Souhaitant étudier l'impact de l'introduction en Bourse sur la conduite stratégique et sur la performance des moyennes entreprises, nous avons porté notre attention sur ces deux derniers mécanismes de contrôle.

Parmi les différentes fonctions des organismes de contrôle et de régulation des marchés financiers, notamment l'AMF (l'autorité des marchés financiers), on retrouve la volonté de protéger les actionnaires et la vérification de la transparence de l'information. De plus, les contraintes de droit ou de fait imposées aux managers par la publication des rapports annuels et lors des différentes réunions avec les actionnaires incitent ces derniers à faire preuve d'une prudence accrue dans les choix stratégiques adoptés. Cependant, ce système est de loin insuffisant pour empêcher les dirigeants de feindre ou de contourner certaines réglementations.

La résolution du conflit actionnaire-gestionnaire passe également par une meilleure redistribution de l'information assurée par le marché financier. Ainsi, la politique financière de la société constitue un signal sur les intérêts des dirigeants. Le choix d'une stratégie plutôt qu'une autre permet aux actionnaires de bénéficier d'une source d'informations dont l'interprétation facilite la compréhension de la politique adoptée par les managers et contribue à la diminution des conflits d'intérêts.

La théorie du signal vise à analyser les mécanismes de fonctionnement des marchés financiers, sachant qu'il existe des problèmes d'asymétrie d'informations entre principal et agent. Elle a pour objet d'expliquer les variations des cours grâce aux signaux émis par les dirigeants à travers leurs décisions stratégiques et leur politique financière. Par exemple, l'endettement constitue un signal positif quant aux flux futurs de l'entreprise. Le dirigeant ne souscrit un nouvel emprunt que s'il est sûr de ses capacités à honorer ses engagements. Le prix de l'action, étant déterminé par l'actualisation des dividendes futurs anticipés, se trouvera alors apprécié. Par ailleurs, l'endettement constitue un mécanisme de résolution des conflits dans la mesure où il incite les dirigeants à être performants pour éviter les risques de faillite et la perte de leur emploi. En effet, plus une entreprise est endettée, plus le risque de défaillance est élevé et plus les dirigeants doivent veiller à ce que leurs décisions maximisent le flux d'activités, de manière à ce que le service de la dette

Revue internationale P.M.E., vol. 18, ${ }^{\text {os }} 3-4,2005$

(C) 2005 - Presses de l'Université du Québec

Édifice Le Delta I, 2875, boul. Laurier, bureau 450, Sainte-Foy, Québec G1V 2M2 • Tél.: (418) 657-4399 - www.puq.ca

Tiré de: Revue internationale P.M.E., vol. 18, nos 3-4, sous la direction de Louis Raymond • PME1803N

Tous droits de reproduction, de traduction et d'adaptation réservés 
puisse être assuré. Du même coup, ils atténuent les risques de perte de leur emploi et maximisent la valeur des actions. Dans le même ordre d'idées, les politiques de dividendes peuvent être interprétées comme des modes de résolution de ces conflits. Rozeff (1982) justifie l'existence de la politique de distribution de dividendes comme moyen de discipliner les dirigeants. En effet, plus une société distribue de dividendes, plus il lui est nécessaire, toutes choses égales d'ailleurs, de recourir au marché financier pour satisfaire ses besoins de financement. L'appel au marché financier constitue en fait un moyen de contrôler la gestion de la firme.

Le marché financier intervient comme ultime système de contrôle à deux niveaux. Premièrement, les actionnaires mécontents ont toujours la possibilité de vendre leurs titres, faisant ainsi baisser la valeur de la société. Cette baisse peut avoir plusieurs conséquences néfastes pour les dirigeants: baisse de leur rémunération s'ils possèdent des actions ou si leur rémunération est indexée sur le cours, baisse de leur valeur sur le marché du travail, difficulté à émettre de nouveaux titres, etc. Deuxièmement, à l'image du marché financier, le marché des fusions-acquisitions constitue un garant de l'efficacité des entreprises. En 1965, Manne notait: «C'est le libre fonctionnement des marchés financiers et de la Bourse ainsi que la concurrence que se livrent les managers pour le contrôle des entreprises qui est le meilleur gage d'un contrôle démocratique» (p. 110-120; traduction libre). Le rôle disciplinaire des prises de contrôle se justifie de plusieurs manières (Derhy, 1996): en premier lieu, la simple menace d'une OPA, qui pèse sur les managers, les contraint à gérer la firme au mieux des intérêts des associés. De plus, cette menace incite les dirigeants à transmettre au marché l'ensemble des informations susceptibles de permettre une évaluation correcte de la firme. Ensuite, les fusions et les acquisitions permettent, grâce au transfert de contrôle, d'éliminer les firmes mal gérées et dont le potentiel de croissance est réel. Aussi les dirigeants peu performants sont-ils souvent écartés de la gestion de la firme après acquisition. Enfin, dans l'hypothèse où l'offre publique se solde par un échec, cela peut conduire les dirigeants en place à remettre en cause leur politique de gestion et à redynamiser les performances de la société.

À la lumière de ce rappel théorique, il apparaît que le marché financier joue un rôle important en matière de gouvernement d'entreprise. Il constitue, selon la typologie de Charreaux (1997), un mécanisme spontané et non spécifique qui contraint les dirigeants à prendre des décisions stratégiques efficaces. Cela est d'autant plus vrai dans le cas des grandes entreprises où la menace d'une OPA est plus pesante du fait de l'atomisation de la structure d'actionnariat. Qu'en est-il du cas particulier des moyennes entreprises, souvent de type familial, avec un actionnariat concentré et un dirigeant-propriétaire? Voici ce qu'en pense Charreaux (1998, p. 111):

Dans le cas des PME, on considère souvent, un peu hâtivement, que la relation actionnaires-dirigeants n'est pas une source de conflits, ce qui est apparemment logique puisqu'il n'y a pas de séparation entre la fonction de propriété et celle de la direction. Pour mieux comprendre le comportement des PME, il faudrait nuancer cette

Revue internationale P.M.E., vol. 18, n ${ }^{\text {os }} 3-4,2005$

(C) 2005 - Presses de l'Université du Québec

Édifice Le Delta I, 2875, boul. Laurier, bureau 450, Sainte-Foy, Québec G1V 2M2 - Tél.: (418) 657-4399 - www.puq.ca

Tiré de: Revue internationale P.M.E., vol. 18 , nos $3-4$, sous la direction de Louis Raymond $\cdot$ PME1803N

Tous droits de reproduction, de traduction et d'adaptation réservés 
conclusion pour tenir compte du fait que les PME sont organisées selon différents statuts juridiques. Les PME ouvrent leur capital à des actionnaires externes, notamment financiers, lorsqu'elles sont introduites sur le Second ou le Nouveau marché; dans ce cas, il y a modification des relations traditionnelles.

Ainsi, nombreuses sont les recherches qui présentent la firme familiale comme étant celle qui engendre les moindres coûts d'agence et qui représente les formes de gouvernance les plus efficientes (Daily et Dolinger, 1992; Kang, 2000). Jensen et Meckling (1976) vont plus loin en considérant qu'il n'est pas nécessaire d'instaurer des mécanismes de gouvernance dans les firmes familiales. Ces dépenses ne sont pas sans réduire leurs performances. Schulz, Lubatkin et Dino (2001) ne partagent pas cet avis. Ils affirment que les dirigeants-propriétaires exposent les firmes au risque moral surtout lorsqu'ils sont libérés de la discipline des marchés (Jensen, 1998). En effet, l'échec du marché financier dans son rôle de contrôle permet aux propriétaires internes (dirigeants) de privilégier leurs propres intérêts au détriment de ceux des autres actionnaires externes (ou minoritaires). L'absence d'un marché liquide accroît la menace de vol ou expropriation. En utilisant le droit de vote et de contrôle des ressources spécifiques de la firme, les dirigeants peuvent spolier les autres actionnaires «pris en otage». Ainsi, les problèmes d'agence peuvent bien exister avec la possibilité d'autocontrôle. Par ailleurs, du fait de l'existence de motivations non pécuniaires, les managers-propriétaires peuvent poser des gestes allant à l'encontre de leurs intérêts économiques. C'est ce qu'appelle Jensen (1998) «agency problems with one self»: «owner managers have incentives to take actions that can harm themselves as well as those around them». Aussi, puisque le pouvoir n'est pas symétriquement distribué dans une firme, le dirigeant-propriétaire peut-il être amené à exploiter ses subordonnés. Perrow (1986, p. 227) parle de l'opportunisme du propriétaire «owner opportunism». Ces firmes sont également exposées à la menace de la sélection adverse due à l'inefficience du marché des cadres dirigeants et de la propriété du capital (absence de possibilité de révocation du dirigeant-propriétaire). Enfin, l'altruisme modifie les structures de motivation des firmes familiales. Par exemple, Simon (1993) et Eshel, Samuelson et Shaked (1998) notent que l'altruisme incite les parents, chefs d'entreprise, à s'occuper prioritairement de leurs enfants, encourage les membres de la famille à prendre en considération les autres et rend les relations familiales très précieuses au point de promouvoir et de soutenir les engagements familiaux au détriment de l'intérêt de la firme. Schulz, Lubatkin et Dino (2001) concluent que les dirigeants-propriétaires ne minimisent pas les coûts d'agence. Il n'en reste pas moins vrai qu'ils croient en l'existence d'une relation positive entre les coûts d'agence engendrés par la firme de type familial et la performance si le système de gouvernement d'entreprise est efficient. Le marché financier en constitue un élément. Quel impact a-t-il sur la stratégie et sur la performance des moyennes entreprises?

Dans cette section, nous avons essayé d'exposer les fondements théoriques qui sous-tendent notre problématique. Nous nous sommes référée aux théories

Revue internationale P.M.E., vol. 18, ${ }^{\text {os }} 3-4,2005$

(C) 2005 - Presses de l'Université du Québec

Édifice Le Delta I, 2875, boul. Laurier, bureau 450, Sainte-Foy, Québec G1V 2M2 • Tél.: (418) 657-4399 - www.puq.ca

Tiré de: Revue internationale P.M.E., vol. 18, nos 3-4, sous la direction de Louis Raymond • PME1803N

Tous droits de reproduction, de traduction et d'adaptation réservés 
de la gouvernance qui considèrent le marché financier comme un mécanisme qui gouverne la conduite des dirigeants et réduit leur latitude managériale. En effet, la contrainte de la transparence et le contrôle continu des choix stratégiques et financiers limitent une conduite opportuniste. Cela s'applique également au cas des PME au sein desquelles la fonction de propriété et celle de décision sont cumulées. A priori, il existerait un lien entre la cotation en Bourse et la conduite stratégique du dirigeant, et ce lien n'est pas sans effet sur la performance. Afin de confirmer (ou d'infirmer) ces propos, nous avons prévu une étude empirique dont la méthodologie est explicitée ci-après. Au préalable, nous justifierons les outils de mesure de performance utilisés.

\section{2. Évaluation de la performance}

L'approche dominante de la performance est celle de la valeur actionnariale dont les fondements sont les suivants. Si on suppose que les marchés sont concurrentiels et efficients, que les dirigeants sont les agents «fidèles» des actionnaires, que ces derniers sont les seuls «créanciers résiduels», alors, il y a confusion entre la maximisation de la richesse des actionnaires et l'efficacité allocative globale. Ces hypothèses, n'étant pas respectées, surtout en raison des externalités, la mesure de la performance à partir de la seule richesse des actionnaires est contestable (Charreaux et Desbrières 1998). Cet avis est loin d'être partagé par tous les auteurs, en particulier par Albouy (1999). Pour ce dernier, la création de valeur pour les actionnaires, en tant que créanciers résiduels, suppose la création de valeur pour les autres partenaires, dans la mesure où l'on peut faire confiance à ces derniers pour défendre leurs intérêts. Une façon d'échapper à ce débat est de supposer que la poursuite de l'objectif de maximisation de la valeur actionnariale conduit in fine à une réduction maximale des pertes d'efficacité et, à terme, à maximiser le bien-être pour l'ensemble des partenaires.

Au-delà des critiques traditionnelles qui peuvent être adressées à l'approche actionnariale, la difficulté d'évaluer la création de valeur pour les sociétés non cotées $^{3}$ oblige à recourir à des mesures comptables ne représentant au mieux que des approximations grossières de la performance. Les mesures comptables occupent une place dominante dans les études existantes. Il n'en reste pas moins vrai qu'elles comportent des limites; celles-ci sont liées aux principes et normes comptables qui ne sont pas uniformes sur le plan international (donc elles se prêtent peu à la

3. Il est, en effet, quasiment impossible d'évaluer le coût des fonds propres des entreprises non cotées. La mesure de la création de valeur par l'EVA (Economic Value Added), le profit économique ou n'importe quel autre indicateur boursier est compromise à partir du moment où l'on ne peut pas déterminer le coût moyen pondéré du capital ni le prix de l'action avant son introduction en Bourse.

Revue internationale P.M.E., vol. 18, $\mathrm{n}^{\text {os }} 3-4,2005$

(C) 2005 - Presses de l'Université du Québec

Édifice Le Delta I, 2875, boul. Laurier, bureau 450, Sainte-Foy, Québec G1V 2M2 - Tél.: (418) 657-4399 - www.puq.ca

Tiré de: Revue internationale P.M.E., vol. 18, nos $3-4$, sous la direction de Louis Raymond - PME1803N

Tous droits de reproduction, de traduction et d'adaptation réservés 
comparaison) et aux possibilités de manipulation ou d'habillage des indicateurs comptables, notamment des résultats. Nous convenons donc que les critères retenus ne sont pas exempts de critiques.

Dans la perspective de la valeur actionnariale, le seul indicateur qui soit fondé, en tant qu'approximation comptable, est le taux de rentabilité des capitaux propres (résultat net/capitaux propres) ${ }^{4}$. L'inconvénient de ce dernier est d'être très sensible aux manipulations comptables, ce qui conduit à lui préférer des indicateurs du type résultat courant/capitaux propres, qui ont l'avantage d'éliminer l'incidence des éléments exceptionnels. Mieux encore, on peut lui substituer des indicateurs de rentabilité économiques du type résultat économique 5 /(capitaux propres plus dettes financières), qui permettent de mesurer la rentabilité obtenue de l'ensemble des capitaux investis. Ces indicateurs, en dépit de leurs limites (biais dans l'information comptable, absence de prise en compte du coût d'opportunité des capitaux investis, non-ajustement pour le risque, etc.), sont les seuls à pouvoir évaluer la performance actionnariale.

Certaines études dont nous nous sommes fortement inspirées ${ }^{6}$ ont recours à une batterie de ratios qui ne constituent pas des indicateurs de rentabilité stricto sensu; certains de ces indicateurs sont a priori disqualifiés pour appréhender la performance au sens traditionnel. Ainsi, Alexandre et Charreaux (2001) utilisent, à part les ratios de rentabilité, des ratios d'activité, des ratios de marge (ou de profitabilité), tels que le résultat net/ventes ou de productivité comme ventes/effectif ou résultat net/effectif, voire des ratios représentant l'effort d'investissement, l'équilibre financier et la politique de dividendes. Ces ratios sont à retenir à titre indicatif. Ils seront plutôt employés comme variables explicatives de l'évolution de la performance après cotation.

La contrainte imposée par la quantification ne doit pas faire perdre de vue le caractère très partiel des approches dominantes de la mesure de la performance. $\mathrm{Si}$, par exemple, on adopte une conception large de la rente organisationnelle ${ }^{7}$,

4. Cet indicateur, très critiqué, n'est pas forcément mauvais. Un certain nombre d'études empiriques (Biddle, Bowen et Wallace, 1997, pour les États-Unis; Pariente, 2000, pour la France) montre qu'il constitue un bon facteur de prévision de la performance boursière réelle au même titre que l'EVA.

5. Le résultat économique correspond approximativement au résultat d'exploitation net d'impôt ou ce qu'on appelle l'EBIT (Earning Before Interest and Taxes).

6. Il s'agit principalement de la méthodologie utilisée par Alexandre et Charreaux (2001).

7. Par exemple, selon l'optique de la valeur partenariale proposée par Charreaux et Desbrières (1998), la valeur partenariale est égale au surplus global, soit la différence entre l'ensemble des produits évalués à leur prix d'opportunité et l'ensemble des coûts des facteurs évalués à leur coût d'opportunité.

Revue internationale P.M.E., vol. 18, nºs 3-4, 2005

(C) 2005 - Presses de l'Université du Québec

Édifice Le Delta I, 2875, boul. Laurier, bureau 450, Sainte-Foy, Québec G1V 2M2 • Tél.: (418) 657-4399 - www.puq.ca

Tiré de: Revue internationale P.M.E., vol. 18, nos $3-4$, sous la direction de Louis Raymond - PME1803N

Tous droits de reproduction, de traduction et d'adaptation réservés 
dont la répartition s'effectue entre les parties prenantes de la firme, les mesures de cette rente effectuées à partir de la seule richesse des actionnaires ne peuvent rendre compte des effets de la cotation sur ce qui revient éventuellement aux clients et aux salariés. Or, il est vraisemblable qu'un des principaux effets de la cotation soit une modification de la répartition de la rente entre les différents partenaires, notamment en faveur des actionnaires qui deviennent plus influents dans un contexte de cotation. De même, la plupart des mesures retenues, étant donné leur caractère statique, ne permettent pas d'évaluer l'incidence de la cotation sur la capacité à créer durablement de la valeur, même si ce dernier aspect peut être appréhendé partiellement, par exemple en étudiant l'évolution d'indicateurs du type investissement en R-D.

Ces considérations ne signifient pas pour autant que les résultats existants sont systématiquement à écarter. Elles conduisent simplement à rappeler, d'une part, que les études quantitatives traditionnelles comportent de nombreuses limites dont il faut tenir compte dans les interprétations et les conclusions et, d'autre part, que les recherches visant à élaborer d'autres indicateurs de performance sont à poursuivre.

\subsection{Constitution de l'échantillon et description des tests}

À l'instar d'Alexandre et Charreaux (2001), nous avons opté pour une étude de nature longitudinale. Pour chacune des entreprises, les données portent sur une période de sept ans (trois ans avant la cotation, l'année de la cotation, et trois ans après). Au départ, nous avons constitué un échantillon de 689 sociétés cotées sur le second marché, sur le nouveau marché et sur le marché libre OTC (marché ouvert à toute cession). Cet échantillon comportait des sociétés de petites et grandes tailles qu'il a fallu retrancher pour ne retenir que les entreprises moyennes. Nous avons ainsi fixé un intervalle de chiffre d'affaires compris entre 5 et 80 millions d'euros à la date d'introduction en Bourse. Un tel critère laisse à désirer vu la variété des secteurs d'activité auxquels appartiennent les entreprises. Une attention minutieuse a été portée à chaque société, compte tenu de son chiffre d'affaires, son effectif et son secteur d'activité. Ainsi, la taille de l'échantillon a été réduite de plus de $80 \%$. La date d'introduction était également un critère déterminant pour le choix des sociétés. Ayant opté pour une étude longitudinale, nous ne pouvions pas travailler sur des sociétés introduites après 1998 (les rapports annuels 2002 n'étant pas tous disponibles), ni avant 1994 (les données très anciennes n'étant pas facilement accessibles). En outre, les sociétés retenues introduites entre 1994 et 1998 et répondant aux exigences relatives à la taille ne mettent pas toutes leurs rapports annuels à disposition. Et comme le centre de documentation de l'autorité des marchés financiers ne fournit que les données des cinq derniers exercices, cela restreint davantage notre échantillon. In fine, l'étude porte sur 38 moyennes entreprises.

Revue internationale P.M.E., vol. 18, n ${ }^{\text {os }} 3-4,2005$

(C) 2005 - Presses de l'Université du Québec

Édifice Le Delta I, 2875, boul. Laurier, bureau 450, Sainte-Foy, Québec G1V 2M2 - Tél.: (418) 657-4399 - www.puq.ca

Tiré de: Revue internationale P.M.E., vol. 18, nos $3-4$, sous la direction de Louis Raymond - PME1803N

Tous droits de reproduction, de traduction et d'adaptation réservés 
L'étude empirique s'est déroulée en deux étapes correspondant aux deux procédures inspirées de l'étude d'Alexandre et Charreaux (2001). La première étape a permis, principalement, de tester l'efficacité statique, en évaluant l'impact de la cotation sur les variables de performance calculées entreprise par entreprise et, en moyenne, sur des périodes de trois ans avant et après cotation. Des tests de différence de médiane (test de Wilcoxon) appliqués aux deux séries de moyennes, calculées avant et après cotation, ont permis de cerner cet impact. Bien qu'au sens strict seuls les indicateurs de rentabilité rendent compte de la performance financière, les autres critères auxquels nous avons eu recours ont également fait l'objet de tests. Cependant, ces autres critères ne sont à considérer qu'à titre complémentaire, comme des éléments permettant de mieux comprendre la formation de la performance, par exemple à travers la politique d'investissement et de financement. De façon à mieux rendre compte de la dynamique temporelle, des tests complémentaires (de Wilcoxon) ont été effectués sur les valeurs des mêmes indicateurs en opposant successivement les années extrêmes $-3 /+3$, puis $-3 / 0$ (période de précotation) et, enfin, $0 /+3$ (période de postcotation). Cette variante de l'étude présente deux avantages. Premièrement, l'effet éventuel de la cotation risque d'être plus accentué et plus apparent s'il est évalué en opposant les données des années -3 et +3 , plutôt qu'à partir des moyennes avant et après cotation. Les changements des niveaux de performance mettent habituellement un certain temps à être visibles et significatifs à travers les données comptables; cette méthode permet de mieux les appréhender. Deuxièmement, cette variante permet de savoir si l'effet s'est produit avant la cotation, après la cotation ou progressivement au cours des sept années d'observations. De cette façon, on peut mettre en évidence, au moins de façon grossière, la dynamique du processus de cotation. Cependant, quelle que soit la méthode retenue, l'effet dynamique de la cotation n'est perçu que très imparfaitement.

Pour répondre à cette critique, nous avons, dans une deuxième étape, eu recours à la procédure qui permet de décrire de façon plus explicite la dynamique temporelle. Il s'agit tout d'abord de mesurer dans un premier modèle de régression (modèle1), les taux d'accroissement de la performance. Pour cela, les principaux indicateurs de performance ont été régressés sur trois variables: 1) le temps T (les valeurs de 1 à 7 désignant les sept années); cette variable permet de prendre en compte l'effet indubitable du temps et de voir si la performance s'améliore au fil des ans; 2) une variable muette $C$ traduisant la cotation (égale 1 si l'entreprise est cotée, 0 sinon): grâce à cette variable nous saurons, a priori, si la cotation est une variable significativement explicative de la performance; et 3) une variable TC égale au produit de la variable «temps» et de la variable muette «cotation». Cette variable nous donnerait une idée de l'évolution de la performance au fil des ans après la cotation. Les conséquences de l'introduction en Bourse ne sont pas instantanées, mais elles s'étalent sur une période plus longue. Compte tenu

Revue internationale P.M.E., vol. 18, nºs 3-4, 2005

(C) 2005 - Presses de l'Université du Québec

Édifice Le Delta I, 2875, boul. Laurier, bureau 450, Sainte-Foy, Québec G1V 2M2 - Tél.: (418) 657-4399 - www.puq.ca

Tiré de: Revue internationale P.M.E., vol. 18, nos $3-4$, sous la direction de Louis Raymond - PME1803N

Tous droits de reproduction, de traduction et d'adaptation réservés 
de la double dimension des données, la régression a porté sur 266 observations (38 entreprises sur sept ans). Les coefficients de la variable $\mathrm{C}$ permettent de mesurer et de tester pour chaque entreprise l'effet de la cotation en seuil. Par son caractère statique, ce test reste très proche dans l'esprit du test présenté dans la première procédure. Il fournit cependant une information sur l'effet de la cotation pour chacune des entreprises. Le coefficient de la variable TC permet de mesurer et de tester l'effet sur l'accroissement de la performance au cours de la cotation, c'est-à-dire sur quatre ans (l'année de la cotation et les trois ans qui suivent), pour chacune des entreprises.

La deuxième phase de cette procédure (modèle 2) consiste à expliquer les mécanismes à travers lesquels la cotation en Bourse influence la performance. Il s'agit de régresser l'accroissement de la performance (les coefficients de TC) sur les variables explicatives de la performance, notamment les variables ayant trait aux décisions stratégiques modifiées à la suite de l'introduction en Bourse. On peut ainsi mesurer l'effet de ces variables sur l'efficacité de la cotation, de façon individuelle, pour chaque firme, plutôt que globalement sur une firme représentative (moyenne) fictive.

\section{Analyse et interprétation des résultats}

\subsection{Les résultats de l'étude statistique: la performance statique}

Le tableau 1 présente les résultats des tests de la première procédure. Pour chaque indicateur sont indiquées les moyennes calculées sur les 38 sociétés étudiées à l'année -3 (c'est-à-dire la troisième année avant introduction); 0 (l'année de l'introduction) $;+3$ (troisième année après introduction) ainsi que la moyenne des trois années avant cotation et après. Les colonnes suivantes font apparaître les tests de Wilcoxon appliqués aux séries de moyennes deux à deux. Ce test nous indique si la différence des moyennes est significative ou pas.

Au vu des ratios de rentabilité, la cotation semble avoir un effet négatif sur la performance. En effet, les deux indicateurs de performance, stricto sensu, soit la rentabilité des capitaux propres (mesurée par le résultat courant ou par le résultat net) et la rentabilité économique, accusent une baisse significative de l'année -3 à l'année +3 : de la moyenne avant cotation à la moyenne après cotation et surtout de l'année 0 à l'année +3 . Cela s'explique par une détérioration des résultats et par une augmentation des capitaux propres (ou des capitaux investis) après la cotation. Nos propos sont confirmés, d'une part, par l'évolution des ratios d'indépendance et d'autonomie financière et, d'autre part, par la chute du taux de marge bénéficiaire. Ce dernier a diminué significativement de l'année -3 à $+3:-3 / 0$ et $0 /+3$.

Revue internationale P.M.E., vol. 18, n ${ }^{\text {os }} 3-4,2005$

(C) 2005 - Presses de l'Université du Québec

Édifice Le Delta I, 2875, boul. Laurier, bureau 450, Sainte-Foy, Québec G1V 2M2 - Tél.: (418) 657-4399 - www.puq.ca

Tiré de: Revue internationale P.M.E., vol. 18, nos $3-4$, sous la direction de Louis Raymond - PME1803N

Tous droits de reproduction, de traduction et d'adaptation réservés 
Certes, la variation du chiffre d' affaires est positive, ce qui signifie un accroissement du chiffre d'affaires d'une année à une autre. Il n'en reste pas moins vrai qu'il se fait à un taux décroissant, c'est pourquoi la variation du chiffre d'affaires a diminué par rapport à la situation d'avant cotation. Notons que cette différence n'est pas significative au vu du test de Wilcoxon. Nous ne pouvons pas en conclure un effet de la cotation sur le niveau d'activité de l'entreprise. Cela dit, le niveau d'exportation s'est accru significativement après l'introduction en Bourse. Probablement parce que l'entreprise dispose de plus de moyens financiers lui permettant d'accroître sa part de marché à l'étranger, ce qui améliore son image pour les investisseurs et reflète des perspectives d'avenir florissant. Par ailleurs, contrairement aux prédictions théoriques, la cotation ne semble avoir aucun effet sur le pouvoir de négociation de l'entreprise avec ses clients et ses fournisseurs, du moins en termes de délai de paiement, ce qui n'empêche pas que l'entreprise puisse négocier les prix (à la hausse pour les clients et à la baisse pour les fournisseurs). Cela n'est cependant pas confirmé au vu de la réduction affichée du ratio EBE/CAHT. Aussi, pour les indicateurs de productivité, le test de Wilcoxon ne permet-il pas de rejeter l'hypothèse nulle d'absence d'effet de la cotation. La seule variation significative est la diminution de la productivité de l'année 0 à +3 . Nous l'expliquons, a priori, par l'accroissement de l'effectif après l'introduction en Bourse.

En ce qui concerne les ratios d'investissement, la cotation semble avoir un effet significatif sur la politique d'investissement de l'entreprise. En effet, son ratio immobilisations/total bilan s'est accru de -3 à +3 , de 0 à +3 et de moyenne avant et après cotation. L'essentiel de son investissement concerne la croissance externe. Le ratio immobilisations financières/total immobilisations n'a pas cessé de croître. Toutefois, les investissements en immobilisations corporelles et incorporelles (notamment la recherche et développement) ont diminué de façon significative. Les dirigeants, semble-t-il, favorisent les stratégies de croissance externe au détriment de la croissance interne. Quant au financement, nous constatons une amélioration significative du ratio d'autonomie et d'indépendance financière entre l'année -3 et 0 . Avant son introduction en Bourse, l'entreprise augmenterait ses capitaux propres pour refléter une santé financière solide et un respect des conditions d'équilibre à long terme $(F R>0)$. Cependant, son niveau d'endettement ne varie pas significativement. Enfin, concernant la politique de dividendes, rares sont les moyennes entreprises qui en distribuent. Dans cette minorité, le taux de distribution s'accroît significativement après l'introduction, et ce, dans un souci de continuité de comportement, de fidélisation des actionnaires et, donc, de maintien de la valeur des actions. En effet, les dirigeants continuent à distribuer des dividendes même en dégageant un résultat déficitaire. Si on suppose que ce ratio témoigne de la discipline exercée par les actionnaires, celle-ci semble se renforcer après la cotation.

$\mathrm{Au}$ total, les variations significatives attribuables à la cotation concernent principalement les ratios de rentabilité, avec une diminution significative après

Revue internationale P.M.E., vol. 18, nºs 3-4, 2005

(C) 2005 - Presses de l'Université du Québec

Édifice Le Delta I, 2875, boul. Laurier, bureau 450, Sainte-Foy, Québec G1V 2M2 • Tél.: (418) 657-4399 - www.puq.ca

Tiré de: Revue internationale P.M.E., vol. 18, nos $3-4$, sous la direction de Louis Raymond - PME1803N

Tous droits de reproduction, de traduction et d'adaptation réservés 
l'introduction en Bourse, les ratios d'investissement, qui témoignent d'une forte croissance externe ${ }^{8}$, et le ratio de distribution, qui illustre le renforcement du pouvoir des actionnaires après la cotation. Ce test, s'il conduit à percevoir plus clairement les effets de la cotation, ne donne cependant qu'une vision grossière de sa dynamique. De plus, appliqué à une firme moyenne fictive, il ne permet pas d'obtenir des résultats individuels, entreprise par entreprise. Pour cette raison, l'étude empirique a été prolongée par une analyse dynamique.

\subsection{Les résultats de l'étude économétrique: la performance dynamique}

L'intégration explicite du temps renouvelle les analyses plus traditionnelles; elle se justifie par le fait qu'il est peu vraisemblable que l'effet de la cotation sur la performance soit immédiat. En effet, les entreprises, même de taille moyenne, sont des systèmes relativement rigides, à forte inertie. Il faut du temps pour que les nouvelles orientations stratégiques ou les modifications organisationnelles, après l'introduction en Bourse, produisent des effets. Ainsi, pour mieux rendre compte de l'effet de la cotation, il faudrait évaluer l'éventuel gain (ou perte) de performance de façon progressive et non pas uniquement à travers un saut quantitatif se produisant à la date de cotation. La procédure que nous avons appliquée comprend deux étapes. Il s'agit tout d'abord (modèle 1) de mesurer le gain (ou la perte) dynamique de performance attribuable à la cotation et de vérifier si la cotation est une variable significativement explicative de la performance. Par la suite, nous avons testé la pertinence des variables pouvant expliquer cette évolution (modèle 2).

\section{Modèle 1: l'évaluation de la performance dynamique}

Le premier modèle de régression, emprunté de l'article d'Alexandre et Charreaux (2001), porte sur les variables de rentabilité. Sa forme générale est la suivante:

$$
\mathrm{Re}_{i t}=\alpha_{i}+\beta_{l i} * \mathrm{~T}_{i t}+\beta_{2 i} * \mathrm{COT}_{i t}+\beta_{3 i} * \mathrm{TC}_{i t}+\beta_{4 i} * \text { taille }_{i t}+\varepsilon_{i t}
$$

$\mathrm{Re}_{i t}$ : la rentabilité économique de la firme $i$ à l'année $t$.

$\mathrm{T}_{i t}$ : le temps pour la firme $i$ (valeur allant de 1 à 7).

$\mathrm{COT}_{i t}$ : une variable muette de cotation prenant la valeur 1 pour l'année $t$ de cotation de l'entreprise $i$, et 0 sinon.

$\mathrm{TC}_{i t}$ : une variable exprimant l'interaction des deux variables précédentes.

8. Faut-il à ce sujet rappeler l'antinomie qui existe entre les objectifs de croissance et de rentabilité? Cela est confirmé dans cette étude, du moins pour la période retenue. À long terme, les deux objectifs devraient converger.

Revue internationale P.M.E., vol. 18, n ${ }^{\text {os }} 3-4,2005$

(C) 2005 - Presses de l'Université du Québec

Édifice Le Delta I, 2875, boul. Laurier, bureau 450, Sainte-Foy, Québec G1V 2M2 • Tél.: (418) 657-4399 - www.puq.ca

Tiré de: Revue internationale P.M.E., vol. 18, nos $3-4$, sous la direction de Louis Raymond P PME1803N

Tous droits de reproduction, de traduction et d'adaptation réservés 


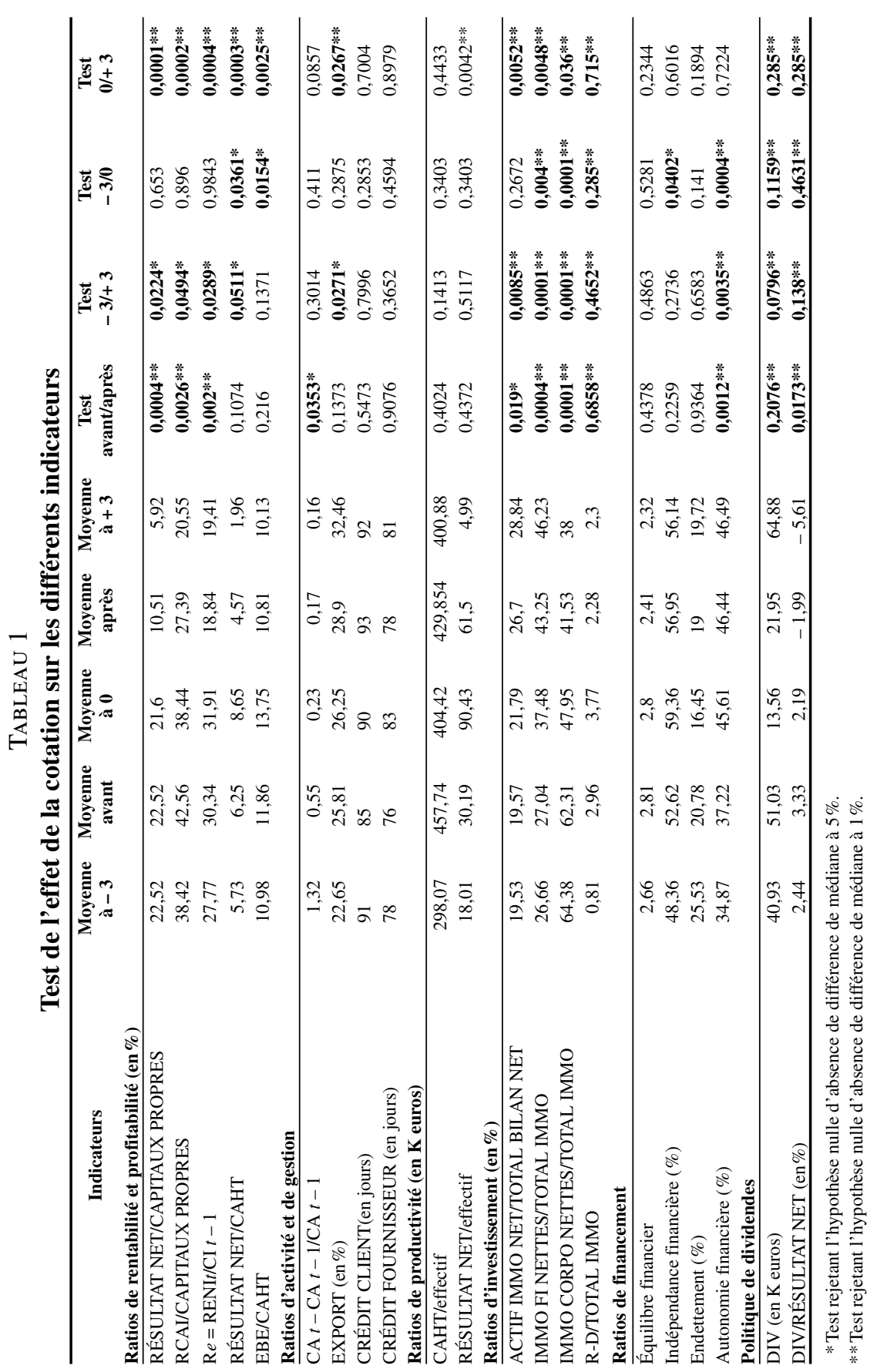

Revue internationale P.M.E., vol. 18, $\mathrm{n}^{\text {os }} 3-4,2005$

(C) 2005 - Presses de l'Université du Québec

Édifice Le Delta I, 2875, boul. Laurier, bureau 450, Sainte-Foy, Québec G1V 2M2 - Tél.: (418) 657-4399 - www.puq.ca

Tiré de: Revue internationale P.M.E., vol. $18, n^{\text {os }} 3-4$, sous la direction de Louis Raymond $\cdot$ PME1803N Tous droits de reproduction, de traduction et d'adaptation réservés 
Taille $_{i t}$ : une variable de contrôle, mesurée par le chiffre d'affaires, de l'entreprise $i$ à l'année $t$.

$\varepsilon_{i t}: \quad$ le terme d'erreur.

Le tableau 2 présente un extrait des résultats obtenus pour la rentabilité économique, variable que nous estimons la plus représentative de la performance. Il s'agit ici d'une régression linéaire sur des données de panel portant sur 266 observations (38 entreprises sur sept ans).

Afin de mieux comprendre le contenu du tableau 2, prenons l'exemple de la société Passat, entreprise pour laquelle tous les coefficients sont significativement différents de 0 . Le coefficient lié à la variable $\mathrm{T}$ est négatif, traduisant en moyenne une baisse de la rentabilité économique sur l'ensemble de ces années. Le coefficient de la variable $\mathrm{C}$, également négatif, représente une baisse de la rentabilité lors de la cotation. Enfin, le coefficient positif de TC signifie que la performance s'améliore au fil des ans après la cotation. En d'autres termes, les perspectives d'amélioration de la performance augmentent après la cotation.

Le coefficient de la variable $C$ fournit une information de nature statique assez proche de celle que les tests de différence de médiane ont fournie. Cependant, dans ce cas, l'information est donnée entreprise par entreprise. L'effet de la cotation est positif pour 21 entreprises sur les 38 de l'échantillon. Toutefois, même si l'effet seuil apparaît positif pour une majorité de sociétés cotées, il n'est positif et significatif que pour deux entreprises seulement.

Cela dit, l'interprétation de $\mathrm{C}$ ne permet que de cerner les effets de seuil, statiques, de la cotation. Pour analyser l'efficacité dynamique, il faut considérer les coefficients de la variable TC. Ils sont positifs pour 18 entreprises sur 38, mais ne sont positifs et significatifs que pour cinq entreprises. En termes d'efficacité dynamique, le gain attribué à la cotation est loin d'être systématiquement confirmé au vu de la performance des firmes de l'échantillon sur la période retenue.

Admettons que l'échantillon considéré est peu représentatif et la période retenue vraisemblablement trop courte pour que les gains d'efficacité dynamique liés à la cotation, s'ils existent, aient eu le temps de se réaliser.

\section{Modèle 2: l'explication des effets dynamiques de la cotation}

Même si les effets dynamiques de la cotation sont rarement significatifs, il reste intéressant, pour mieux les comprendre, de tester l'influence des variables stratégiques, financières et de gouvernance sur la performance, en proposant un second modèle de régression dont elle constitue la variable dépendante. 


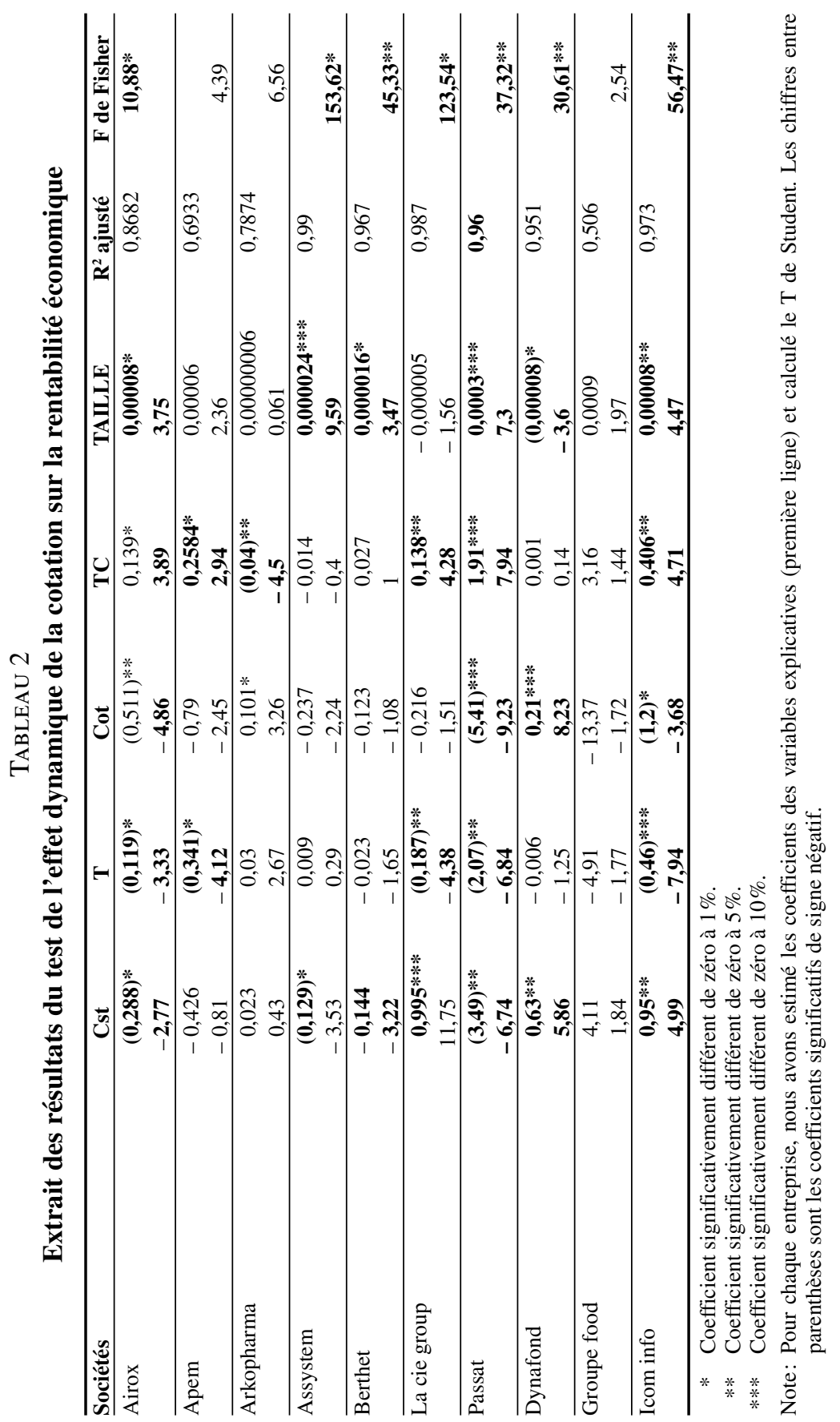

Revue internationale P.M.E., vol. 18, n $3-4,2005$

(C) 2005 - Presses de l'Université du Québec

Édifice Le Delta I, 2875, boul. Laurier, bureau 450, Sainte-Foy, Québec G1V 2M2 • Tél.: (418) 657-4399 - www.puq.ca

Tiré de: Revue internationale P.M.E., vol. 18, nos $3-4$, sous la direction de Louis Raymond - PME1803N Tous droits de reproduction, de traduction et d'adaptation réservés 
Nous avons retenu comme variables explicatives de l'évolution de la performance après cotation des variables stratégiques pouvant être affectées à la suite de l'introduction en Bourse. Il s'agit du niveau de croissance interne ou CI mesuré par le ratio (immobilisations corporelles/total immobilisations); du niveau de croissance externe ou CE (immobilisations financières/total immobilisations); et des efforts en recherche et développement ( $\mathrm{R}-\mathrm{D} /$ total immobilisations). Des indicateurs de stratégies concurrentielles ont été partiellement pris en compte à travers l'évaluation du pouvoir de négociation avec les clients ou PNCLT (mesuré en termes de délai de paiement), du pouvoir de négociation des fournisseurs ou PNFR et de l'évolution de la part de marché à l'étranger ( $\mathrm{X}$ : pourcentage des exportations).

La structure financière constitue également un facteur explicatif de la performance. Rappelons à ce sujet les propositions des auteurs classiques en finance, notamment celles de Modigliani et Miller (1963) et Miller (1977). Vraisemblablement, l'introduction en Bourse offre la possibilité de recourir davantage à l'augmentation du capital (CAPP). Cela signifie, toutes choses égales d'ailleurs, une baisse relative de l'endettement $(\mathrm{DF})^{9}$. En ce qui concerne la gouvernance, un tel résultat pourrait traduire un effet de substitution entre les disciplines exercées respectivement par les créanciers et par les actionnaires.

Dans le même ordre d'idées, toujours en ce qui concerne la gouvernance, la cotation est censée impliquer une discipline nouvelle imposée par le marché financier ${ }^{10}$, une reconfiguration de l'actionnariat et un renforcement du pouvoir des actionnaires. Nous retiendrons, de ce fait, le taux de distribution des dividendes (DIV), la géographie du capital (FAMI, MANA) ${ }^{11}$ et le compartiment du marché boursier (COMP2, COMP3) comme variables explicatives.

9. Cela est confirmé au vu de la matrice de corrélation. Le coefficient est négatif et très élevé.

10. Il est clair que les contraintes imposées aux entreprises cotées diffèrent selon leur marché de cotation. Les exigences du second marché ne sont pas les mêmes que celles du nouveau marché et encore moins celles du marché libre. C'est la raison pour laquelle nous avons introduit dans notre modèle le compartiment boursier dans lequel chaque entreprise est cotée.

11. Selon Charreaux et Pitol-Belin (1985) et Charreaux (1997), dans les sociétés cotées françaises, il existe trois types de société en fonction de leur structure de propriété: 1) les firmes managériales: aucun dirigeant ne détient une part significative du capital; 2) les firmes familiales: une famille détient la majeure partie du capital et nomme un de ses membres pour diriger; 3 ) les firmes contrôlées: le capital est concentré; les représentants de la firme (ou des firmes) qui contrôle dominent le conseil d'administration et nomment les dirigeants.

Cette typologie sera utilisée pour étudier l'influence de la structure de propriété sur l'évolution de la performance après cotation. Il semble plus adapté de partir d'une typologie fondée sur l'élément central de la théorie, à savoir la relation d'agence entre dirigeants et actionnaires, plutôt que de centrer l'analyse sur le pourcentage de capital détenu par les dirigeants.

Revue internationale P.M.E., vol. 18, n ${ }^{\text {os }} 3-4,2005$

(C) 2005 - Presses de l'Université du Québec

Édifice Le Delta I, 2875, boul. Laurier, bureau 450, Sainte-Foy, Québec G1V 2M2 - Tél.: (418) 657-4399 - www.puq.ca

Tiré de: Revue internationale P.M.E., vol. 18, nos $3-4$, sous la direction de Louis Raymond P PME1803N

Tous droits de reproduction, de traduction et d'adaptation réservés 
Enfin, l'amélioration de la performance après la cotation est vraisemblablement conditionnée par la complexité de l'entreprise et son niveau de performance initial. Plus l'entreprise est complexe ou plus son niveau de performance initiale est faible, plus il sera long et difficile de mener à bien le processus de cotation et d'obtenir des gains de performance. Pour rendre compte de ce phénomène, nous avons introduit l'effectif (EFF) comme variable, certes discutable, de la complexité et le niveau de performance initiale (PERFI) mesurée par la rentabilité économique à la date d'introduction en Bourse.

Bref, sans faire une étude exhaustive des processus par lesquels la cotation influence la performance, objectif auquel ne peut prétendre cette recherche et qui est vraisemblablement au-delà des possibilités ouvertes par les études économétriques, on peut cependant poser l'hypothèse que la cotation a un effet sur la performance des moyennes entreprises en modifiant leurs données stratégiques. Cette modification s'appréhende à travers les dimensions suivantes: la possibilité de s'internationaliser, de réaliser des augmentations de capital par appel public à l'épargne et celle de recourir davantage à la croissance...

Le modèle 2 s'écrit de la manière suivante:

$$
\operatorname{VARPERF}_{i}=\alpha+\beta_{k} \mathrm{~V}_{i k}+\varepsilon_{i}
$$

Avec:

VARPERF $_{i}$ : la valeur de la pente de la variable TC dans le modèle (1) pour la firme $i$.

$\mathrm{V}_{i k}$ : la valeur de la $k^{\mathrm{e}}$ variable explicative pour l'entreprise $i$.

$\beta_{k}$ : $\quad$ le coefficient associé à la $k^{\mathrm{e}}$ variable explicative.

Afin d'améliorer la qualité du modèle, un premier travail exploratoire nous a amenée à éliminer certaines variables explicatives, soit parce qu'elles n'étaient pas significatives (tels le niveau de performance initiale, le taux de distribution des dividendes, les compartiments du marché boursier, le taux d'exportation et la structure d'actionnariat), soit parce qu'elles entraînaient de sérieux problèmes de corrélation (c'est le cas des variables croissance interne, pouvoir de négociation des clients, niveau des capitaux propres).

Après avoir éliminé l'essentiel des problèmes posés par la multicolinéarité, le modèle suivant (tableau 3) semble pouvoir être accepté.

Selon ce modèle, l' augmentation de la rentabilité économique (RE) après la cotation dépendrait de façon significative du niveau d'endettement et du pouvoir de négociation du fournisseur.

Revue internationale P.M.E., vol. 18, nos 3-4, 2005

(C) 2005 - Presses de l'Université du Québec

Édifice Le Delta I, 2875, boul. Laurier, bureau 450, Sainte-Foy, Québec G1V 2M2 • Tél.: (418) 657-4399 - www.puq.ca

Tiré de: Revue internationale P.M.E., vol. 18 , nos $3-4$, sous la direction de Louis Raymond $\cdot$ PME1803N

Tous droits de reproduction, de traduction et d'adaptation réservés 
TABLEAU 3

Résultat final des mécanismes d'impact de la cotation sur l'évolution de la performance

Variable dépendante: VARPERF

Méthode: Moindre carré

Échantillon: 138

Observations incluses: 38

\begin{tabular}{lrlcc}
\hline \multicolumn{1}{c}{ Variable } & Coefficient & Std. Error & t-Statistic & Prob. \\
\hline $\mathrm{C}$ & 1,263477 & 0,454419 & 2,780422 & 0,0090 \\
$\mathrm{CE}$ & 0,234819 & 0,451812 & 0,519727 & 0,6068 \\
$\mathrm{DF}$ & $-0,018253$ & 0,010789 & $-1,691797$ & 0,1004 \\
$\mathrm{EFF}$ & $-0,001516$ & 0,001138 & $-1,331725$ & 0,1924 \\
PNFR & $-0,011473$ & 0,005192 & $-2,209952$ & 0,0344 \\
$\mathrm{R} \_D 01$ & 2,377881 & 2,079616 & 1,143423 & 0,2613 \\
\hline $\mathrm{R}^{2}$ & 0,269737 & Moyenne de la variable dépendante & $-0,029000$ \\
$\mathrm{R}^{2}$ ajusté & 0,155633 & Écart type de la variable dépendante & 0,921067 \\
Erreur Standard de la reg. & 0,846363 & Critère d'information d'Akaike & 2,648202 \\
Somme des carrés des & 22,92257 & Critère de Schwarz & 2,906769 \\
résidus & & & \\
Log likelihood & $-44,31584$ & Statistique de Fischer & 2,363962 \\
Statistique Durbin-Watson & 1,830046 & Probabilité (statistique de Fischer) & 0,062005 \\
\hline
\end{tabular}

Le coefficient associé à l'endettement est négatif, ce qui signifie que la variation de la rentabilité économique après la cotation est négative lorsque le niveau d'endettement augmente. Ce résultat n'a rien de surprenant, puisque la rentabilité économique varie inversement avec le niveau des capitaux investis (capitaux propres plus dettes financières). D'autre part, l'effet de levier n'apparaît pas au niveau de la rentabilité économique (RE). Cette dernière dépend exclusivement de l'exploitation. Précisons que les charges financières et les économies d'impôt en découlant ne sont pas prises en compte dans ce ratio. Nous pouvons, de plus, citer le caractère risqué de la dette qui restreint le champ d'action du dirigeant au plan de ses choix stratégiques (notamment de croissance). A priori, un niveau d'endettement élevé incite les dirigeants à faire des investissements moins risqués et donc moins rentables.

Par ailleurs, concernant le pouvoir de négociation des fournisseurs, cette variable semble avoir un effet significatif sur l'évolution de la performance après la cotation. En effet, plus le pouvoir de négociation des fournisseurs est élevé, pour une raison ou une autre, plus les délais de paiement sont courts et plus encore les prix sont élevés. Cela accroît le montant des charges et affecte donc le résultat d'exploitation et la rentabilité de l'entreprise.

La variable «effectif» présente également un signe négatif, ce qui signifie que la performance se détériore avec l'accroissement de l'effectif et avec la complexité 
organisationnelle qui en découle. Cet effet, même non significatif, semble évident, puisque les charges de personnels compressent l'EBE. Cela est d'autant plus vrai que la productivité ne s'améliore pas.

Les coefficients de la variable croissance externe et R-D, quant à eux, sont positifs. La rentabilité économique s'améliore avec de tels investissements. En revanche, ce qui nous paraît contre-intuitif, c'est l'effet non significatif de ces variables qui sont tout de même déterminantes de la performance. Nous expliquons cela par la période retenue. En effet, trois ans ne suffisent pas pour évaluer les conséquences d'un investissement dont les retombées ne se verront qu'à long terme.

Enfin, notons que ce modèle, même s'il nous a permis d'aboutir à quelques conclusions plus ou moins proches de la théorie, demeure une approximation grossière de la réalité qui n'est pas exempte de critiques. En effet, seulement deux variables sur cinq sont significatives. Il est vrai que l'effet significatif de l'ensemble des variables est assez fort, ne présentant qu' un taux d'erreur de $6 \%$ au vu du test de Fisher. Cela n'empêche pas que le $\mathrm{R}^{2}$ ajusté demeure faible. L'évolution de la performance après cotation n'est expliquée qu'à raison de $15 \%$ par les variables retenues. Il conviendrait de creuser davantage pour dégager d'autres facteurs explicatifs. Il serait également intéressant d'intégrer des variables contextuelles qui échappent au contrôle des firmes mais qui peuvent indirectement influencer leur performance; nous pensons particulièrement au cycle économique et au taux de croissance du PIB.

\section{Conclusion}

Au terme de ces développements, pouvons-nous conclure que l'introduction en Bourse affecte la conduite stratégique dans le sens de l'amélioration de la performance des moyennes entreprises?

Au premier abord, l'examen sommaire des principaux indicateurs effectués à partir des moyennes laisse imaginer une baisse substantielle de la rentabilité des capitaux propres et des capitaux investis (RE). Par ailleurs, les entreprises cotées, après leur introduction en Bourse, se sont plus orientées vers les stratégies de croissance externe et leur équilibre financier était toujours maintenu. Si le pouvoir de négociation avec leurs partenaires peut être considéré comme stable en moyenne, la part de l'activité à l'étranger s'est accrue. Enfin, les actionnaires se sont vu attribuer une part supérieure de dividendes. Un examen plus approfondi et plus critique de ces résultats montre, cependant, qu'il n'est pas sûr que ces évolutions, même significatives, soient systématiquement attribuables au phénomène de cotation. Nous avons été amenés à utiliser un modèle économétrique pour tester l'effet significatif de cette variable et voir si, effectivement, elle influence la rentabilité des entreprises et dans quel sens.

Revue internationale P.M.E., vol. 18, nºs 3-4, 2005

(C) 2005 - Presses de l'Université du Québec

Édifice Le Delta I, 2875, boul. Laurier, bureau 450, Sainte-Foy, Québec G1V 2M2 • Tél.: (418) 657-4399 - www.puq.ca

Tiré de: Revue internationale P.M.E., vol. 18 , nos $3-4$, sous la direction de Louis Raymond $\cdot$ PME1803N

Tous droits de reproduction, de traduction et d'adaptation réservés 
Nous avons trouvé que la cotation n'a eu un effet favorable sur la performance que pour une très faible minorité de moyennes entreprises. Le plus souvent, cet effet n'est pas significatif et s'il l'est, il conduit autant à conclure que la cotation entraîne une perte de performance que l'inverse. De plus, les résultats obtenus par le modèle explicatif de l'évolution de la performance après cotation (modèle 2) conduisent à des conclusions peu conformes aux prédictions théoriques.

Ces résultats, très nuancés, doivent-ils amener à contester le bien-fondé de la décision d'introduction en Bourse et à rejeter les justifications traditionnelles de la cotation assises, en particulier, sur les théories de la gouvernance? Nous ne le pensons pas!

Nous convenons parfaitement que la période retenue est très courte pour permettre d'évaluer l'effet de la cotation sur la conduite stratégique des dirigeants. Certes, les stratégies de croissance ont été mises en évidence, mais leurs conséquences ne s'observeront qu'à plus long terme. De plus, les mesures de performance retenues et les outils d'analyse ne sont pas épargnés par les critiques. Ce n'est peut-être pas tant la pertinence de la décision d'introduction en Bourse et les théories qui la fondent qui doivent être soupçonnées que les instruments utilisés pour en mesurer les effets.

L'ambiguïté des résultats semble donc mener à rechercher dans les méthodes employées pour mesurer l'effet de la cotation sur la performance. Il semble en particulier que les études quantitatives traditionnelles aient des difficultés à rendre compte de cet effet. Outre les problèmes de pertinence et de fiabilité des mesures de performance, ces études se heurtent à de multiples difficultés liées à la complexité du processus de cotation, à sa dimension temporelle qui dépasse souvent les périodes retenues et à son caractère contingent, par rapport au contexte économicopolitique et aux données de l'entreprise elle-même. L'imbrication des variables et l'existence vraisemblable d'effets de seuil ou, plus généralement, de non-linéarité, même s'il existe des méthodes pour corriger ou prendre en compte ces effets, incitent à n'accorder qu'une confiance limitée aux résultats obtenus par notre étude économétrique.

Au vu de ces critiques, il semble que pour mieux comprendre les effets de la cotation sur la performance, il faille, au moins de façon complémentaire, recourir à des études cliniques qualitatives. De telles études, d'utilisation courante dans certains champs de la théorie des organisations, peuvent vraisemblablement permettre de mieux cerner l'incidence des effets contextuels et de mieux comprendre comment les modifications des systèmes de gouvernance et les adaptations de l'architecture organisationnelle associées à la cotation en Bourse permettent d'améliorer la performance. 


\section{Bibliographie}

Albouy, M. (1999), «Théorie, applications et limites de la mesure de la création de valeur», Revue française de gestion, $\mathrm{n}^{\circ} 122$, p. 81-90.

Alexandre, H. et G. Charreaux (2001), «Les privatisations françaises ont-elles été efficaces?», Association française de finance, juin, p. 1-76.

BidDle, G.C, R.M. Bowen et J.S. Wallace (1997), «Does EVA beat earnings? Evidence on associations with stock returns and firm values», Journal of Accounting and Economics, vol. 24.

Castanias, R.P. et C.E. Helfat (1992), «Managerial and windfall rents in the market for corporate control», Journal of Economic Behavior and Organization, ${ }^{\circ} 18$, p. 153-184.

Charreaux, G. (1997), Le gouvernement des entreprises: théories et faits, Paris, Economica, $540 \mathrm{p}$.

Charreaux, G. (1998), «Gouvernance des PME-PMI», dans PME-PMI: le métier de dirigeant et son rôle d'agent de changement, Paris, Economica, p. 109-116.

Charreaux, G. et P. Desbrières (1998), «Gouvernement des entreprises et création de valeur», Actes des XIV Journées nationales des IAE, Nantes.

Charreaux, G. et J.P. Pitol-Belin (1985), «La théorie contractuelle des organisations, une application au conseil d'administration», Économies et Sociétés, Séries Sciences de gestion, $\mathrm{n}^{\circ}$ 6, p. 149-181.

Daigne, J.-F. et X. Joly (1986), Le second marché: un atout pour l'entreprise, Paris, Les Éditions d'Organisation, p. 5-75.

DAILy, C.M. et M.J. Dolinger (1992), «An empirical examination of ownership structure in family and professionally-managed firms », Family Business Review, vol. 5, n 2, p. 117-136.

DERHY, A. (1996), Les opérations de fusion-acquisition: analyse théorique et application au marché français, Thèse de doctorat, Université de Paris I, Panthéon-Sorbonne.

Desjardin, C. (1999), «S'introduire en Bourse: combien ça coûte?», Option Finance, n $^{\circ} 565$, p. $12-17$.

Desroches, J. et V. Jog (1991), La PME \& le financement public, Montréal, Institut de recherches politiques, p. 110.

Eshel, I., L. SAmuelson et A. Shaked (1998), «Altruists, egoists and holligans in a local interaction model», American Economic Review, vol. 88, n 1, p. 157-179.

Grossman, S. et O. Hart (1981), «The allocational role of takeover bids in situation of asymmetric information», Journal of Finance, vol. 36, n 2, p. 253-270.

JENSEN, M.C. (1993), «The modern industrial revolution, exit, and the failure of internal control systems», Journal of Finance, vol. 18, n 3, p. 830-880.

Jensen, M.C. (1998), Self Interest, Altruism, Incentives, and Agency, Foundations of Organizational Strategy, Cambridge, Mass., Harvard University Press.

Revue internationale P.M.E., vol. 18, nos 3-4, 2005

(C) 2005 - Presses de l'Université du Québec

Édifice Le Delta I, 2875, boul. Laurier, bureau 450, Sainte-Foy, Québec G1V 2M2 • Tél.: (418) 657-4399 - www.puq.ca

Tiré de: Revue internationale P.M.E., vol. 18, nos $3-4$, sous la direction de Louis Raymond - PME1803N

Tous droits de reproduction, de traduction et d'adaptation réservés 
JENSEN, M.C. et W.H. MecKLING (1976) «Theory of the firm: managerial behavior, agency costs and owner structure», Journal of Financial Economics, vol. 3, p. 305-360.

KANG, D. (2000), The Impact of Family Ownership on Performance in Public Organisations: A Study of the US Fortune 500, 1982-1994, Toronto, Academy of Management Meetings.

Levasseur, M. et A. Quintart (1998), «L'entreprise et la Bourse», dans Finance, Paris, Economica, p. 609-636.

Manne, H.G. (1965), «Mergers and the market for corporate control», Journal of Political Economy, avril, p. 110-120.

MaY, D.O. (1995), «Do managerial motives influence firm risk reduction strategies?», Journal of Finance, vol. 50, no 4, p. 1291-1308.

Miller, M. (1977), «Debt and taxes», Journal of Finance, $n^{\circ}$ 1, p. 337-347.

Modigliani, F. et M. Miller (1963), «Corporate income taxes and the cost of capital: a correction», American Economic Review, n² 2, p. 433-443.

PACQuerot, M. (1997), «Stratégie d'enracinement des dirigeants, performance de la firme et structures de contrôle», dans G. Charreaux (dir.), Le gouvernement des entreprises: théories et faits, Paris, Economica, p. 105-138.

PARIEnTe, S. (2000), «Rendement boursier, création de valeur et données comptables: une étude sur le marché français», Finance Contrôle Stratégie, vol. 3, n 3, p. 125-153.

Perrow, C. (1986), Complex Organisations, New York, Random House.

Ross, S.A. (1973), «The economic theory of agency: the principal's problem», American Economic Review, vol. 63, n 2, p. 134-139.

RozeFF, M.S. (1982), «Growth, bests and agency costs as determinants of dividend payout ratios», Journal of Financial Research, vol. 5, p. 249-259.

SAADA, C. (1996), «L'entreprise et la Bourse», Cahiers français, n 27, p. 73-80.

SChulz, W., M. LubatKIn et R. Dino (2001), «Agency relationships in family firms: theory and evidence», Organisation Science, vol. 12, n 2, p. 99-116.

Shleifer, A. et R.W. Vishny (1989), «Management entrenchment: the case of manager specific investments », Journal of Financial Economics, vol. 25, p. 123-139.

Simon, H.A. (1993), «Altruism and economics», American Economic Review, n 83, p. 156-161. 